\title{
AN INEQUALITY CONCERNING THREE FUNDAMENTAL DIMENSIONS OF PARACOMPACT $\sigma$-SPACES
}

\author{
SHINPEI OKA
}

Abstract. It is shown that Ind $X<\operatorname{dim} X+$ ind $X$ for any nonempty paracompact $\sigma$-space.

In this paper a map is a continuous map and a space is a Hausdorff topological space. The symbols dim, Ind and ind usually denote covering dimension, large inductive dimension and small inductive dimension, respectively. As is well known, ind $X \leqslant$ Ind $X$ and $\operatorname{dim} X \leqslant$ Ind $X$ for any normal space $X$. A space is called a $\sigma$-space if it admits a $\sigma$-locally finite net (cf. [7]). The aim of this paper is to prove the following inequality.

THEOREM 1. Let $X$ be a nonempty paracompact $\sigma$-space. Then Ind $X<\operatorname{dim} X+$ ind $X$.

COROllary 2. Let $X$ be a paracompact $\sigma$-space with ind $X<0$. Then $\operatorname{dim} X=$ Ind $X$.

Our arguments are based on Leibo's ideas of constructing a special kind of map onto metric spaces and making use of Pasynkov's factorization theorem (see [3]).

The following lemma is implicit in [3], and is generalized in [5] to the case where $\mathcal{Q}$ is $\sigma$-discrete and in [6] to the case where $\mathcal{Q}$ is $\sigma$-locally finite.

LEMMA 3. Let $X$ be a submetrizable space (i.e. $X$ admits a weaker metric topology), and let $\mathcal{Q}$ be a countable collection of cozero sets of $X$. Then there exist a metric space $M$ and a one-to-one map from $X$ onto $M$ such that $f(U)$ is an open set of $M$ for every $U \in \mathcal{Q}$.

The following lemma is well known (see [2, Lemma 2.3.16]).

LEMma 4. Let $X$ be a space and let $C, K$ be disjoint closed sets of $X$. Let $थ$ be a countable open cover of $X$ such that, for each $U \in \mathcal{Q}$, either $\bar{U} \cap C=\varnothing$ or $\bar{U} \cap K=\varnothing$. Then $C$ and $K$ are separated by a closed set $S$ such that $S \subset$ $\cup\{\operatorname{Bd} U: U \in \mathcal{U}\}$.

Let $X$ be a paracompact $\sigma$-space. Let $\mathscr{F}=\cup_{i=1}^{\infty} \mathscr{F}_{i}$ and $\mathscr{V}=\cup_{i=1}^{\infty} \mathcal{V}_{i}$ be collections of subsets of $X$ satisfying the following conditions:

(1) $\mathcal{F}$ consists of closed sets of $X$, and $\mathscr{V}$ consists of open sets of $X$;

Received by the editors February 16, 1981.

1980 Mathematics Subject Classification. Primary 54F45, 54E18.

Key words and phrases. $\sigma$-space, covering dimension, large inductive dimension, small inductive dimension. 
(2) $\mathcal{F}$ is a net of $X$;

(3) for each $i, \mathfrak{V}_{i}$ is written as $\mathfrak{V}_{i}=\left\{V_{i}(F): F \in \mathscr{F}_{i}\right\}$, and $F \subset V_{i}(F)$ for each $F \in \mathscr{F}_{i}$;

(4) $\mathfrak{V}_{i}$ is discrete in $X$ for each $i$.

Every paracompact $\sigma$-space admits such collections. For each $i$, put

$$
F_{i}=\cup\left\{F: F \in \mathscr{F}_{i}\right\} \text { and } V_{i}=\cup\left\{V: V \in \mathscr{V}_{i}\right\}
$$

LEMMA 5. Let $X$ be a nonempty paracompact $\sigma$-space and let $\mathcal{F}, \mathcal{V}$ be collections of subsets of $X$ satisfying (1)-(4) above. Let $Y$ be a totally normal space and let $f: X \rightarrow Y$ be a one-to-one onto map such that, for each $i, f\left(F_{i}\right)$ is a closed set and $f\left(V_{i}\right)$ is an open set of $Y$. Then Ind $X<$ Ind $Y+$ ind $X$.

Proof. The proof is by induction on Ind $Y+$ ind $X$. Suppose Ind $Y+$ ind $X=$ 0 . Let $C$ and $K$ be disjoint closed sets of $X$. For each $i$, put $\mathscr{F}_{i}(C)=\left\{F \in \mathscr{F}_{i}: F \subset\right.$ $W$ for some clopen set $W$ with $W \cap C=\varnothing$. For each $F \in \mathscr{F}_{i}(C)$, fix such a $W$ and denote it by $W_{i}(F, C)$. Since ind $X=0$, it follows from (2) that $\cup_{i=1}^{\infty} \mathscr{F}_{i}(C)$ covers $X-C$. Since Ind $Y=0$, it follows from assumption that, for each $i$, there exists a clopen set $O_{i}$ of $Y$ such that $f\left(F_{i}\right) \subset O_{i} \subset f\left(V_{i}\right)$. By (4), $V_{i}(F) \cap f^{-1}\left(O_{i}\right)$ is a clopen set for each $F \in \mathscr{F}_{i}$. Now put

$$
H_{i}(C)=\cup\left\{W_{i}(F, C) \cap V_{i}(F) \cap f^{-1}\left(O_{i}\right): F \in \mathscr{F}_{i}(C)\right\} .
$$

Then, by (4) again, $H_{i}(C)$ is a clopen set of $X$. Clearly $X-C=\cup_{i=1}^{\infty} H_{i}(C)$. Similarly we can find clopen sets $H_{i}(K), i=1,2, \ldots$, such that $X-K=$ $\bigcup_{i=1}^{\infty} H_{i}(K)$. Hence, by Lemma $4, C$ and $K$ are separated by the empty set and, therefore, Ind $X=0$.

Now suppose that the lemma is valid when Ind $Y+$ ind $X<n-1$, and consider the case when Ind $Y+$ ind $X=n$. Let $A$ and $B$ be disjoint closed sets of $X$. For each $i$, put $\mathscr{F}_{i}(A)=\left\{F \in \mathscr{F}_{i}: F \subset W\right.$ for some open set $W$ with $\bar{W} \cap A=\varnothing$ and ind Bd $W \leqslant$ ind $X-1\}$. For each $F \in \mathscr{F}_{i}(A)$, fix such a $W$ and denote it by $W_{i}(F, A)$. By (2), $\cup_{i=1}^{\infty} \mathscr{F}_{i}(A)$ covers $X-A$. Note that for any $X^{\prime} \subset X, f \mid X^{\prime}: X^{\prime} \rightarrow$ $f\left(X^{\prime}\right)$ satisfies the assumption of Lemma 5 with respect to $\mathscr{F} \mid X^{\prime}$ and $\mathfrak{V} \mid X^{\prime}$. Since

Ind $f\left(\operatorname{Bd} W_{i}(F, A)\right)+$ ind $\operatorname{Bd} W_{i}(F, A) \leqslant$ Ind $Y+($ ind $X-1)=n-1$,

it follows from the induction hypothesis that Ind $\mathrm{Bd} W_{i}(F, A)<n-1$. On the other hand, by assumption, we can find an open set $O_{i}$ of $Y$ such that $f\left(F_{i}\right) \subset O_{i} \subset$ $\bar{O}_{i} \subset f\left(V_{i}\right)$ and Ind $\mathrm{Bd} O_{i} \leqslant \operatorname{Ind} Y-1$. Since Ind $\mathrm{Bd} O_{i}+\operatorname{ind} f^{-1}\left(\operatorname{Bd} O_{i}\right)<$ Ind $Y-1+$ ind $X=n-1$, it follows from the induction hypothesis that Ind $\mathrm{Bd} f^{-1}\left(O_{i}\right) \leqslant$ Ind $f^{-1}\left(\operatorname{Bd} O_{i}\right)<n-1$. By (4), $\operatorname{Bd}\left(V_{i}(F) \cap f^{-1}\left(O_{i}\right)\right) \subset$ $\operatorname{Bd} f^{-1}\left(O_{i}\right)$. Now put

$$
H_{i}(A)=\cup\left\{W_{i}(F, A) \cap V_{i}(F) \cap f^{-1}\left(O_{i}\right): F \in \mathscr{F}_{i}(A)\right\} .
$$

Since

$$
\begin{aligned}
\text { Ind } \operatorname{Bd}( & \left.W_{i}(F, A) \cap V_{i}(F) \cap f^{-1}\left(O_{i}\right)\right) \\
& \leqslant \max \left\{\text { Ind Bd } W_{i}(F, A), \text { Ind } \operatorname{Bd}\left(V_{i}(F) \cap \mathrm{f}^{-1}\left(O_{i}\right)\right)\right\}<n-1,
\end{aligned}
$$


it follows from (4) that Ind Bd $H_{i}(A) \leqslant n-1$. Clearly $X-A=\cup_{i=1}^{\infty} H_{i}(A)$ and $A \cap \mathrm{Cl} H_{i}(A)=\varnothing$ for each $i$. Similarly there exist open sets $H_{i}(B), i=1,2, \ldots$, such that Ind $\mathrm{Bd} H_{i}(B) \leqslant n-1, X-B=\cup_{i=1}^{\infty} H_{i}(B)$ and $B \cap \mathrm{Cl} H_{i}(B)=\varnothing$ for each $i$. Then, applying Lemma 4, we have a closed set $S$ separating $A$ and $B$ such that $S \subset\left(\cup_{i=1}^{\infty} \mathrm{Bd} H_{i}(A)\right) \cup\left(\cup_{i=1}^{\infty} \mathrm{Bd} H_{i}(B)\right)$. By the countable sum theorem for Ind, we have Ind $S \leqslant n-1$. Thus Ind $X \leqslant n$, which completes the proof of Lemma 5.

Proof of TheOrem 1. Let $X$ be a nonempty paracompact $\sigma$-space, and let $\mathscr{F}=\cup_{i=1}^{\infty} \mathscr{F}_{i}$ and $\mathfrak{V}=\cup_{i=1}^{\infty} \mathscr{V}_{i}$ be collections of subsets of $X$ satisfying (1)-(4) above. By [1, Lemma 8.2] and [7, Theorem 4.6], $X$ is submetrizable. Thus Lemma 3 applies to yield a metric space $L$ and a one-to-one onto map $g: X \rightarrow L$ such that, for each $i, g\left(F_{i}\right)$ is a closed set and $g\left(V_{i}\right)$ is an open set of $L$. By Pasynkov's factorization theorem [8, Theorem 29], there exist a metric space $M$ and onto maps $f: X \rightarrow M$ and $h: M \rightarrow L$ such that $g=h \circ f$ and $\operatorname{dim} M \leqslant \operatorname{dim} X$. Clearly $f$ is one-to-one, $f\left(F_{i}\right)$ is a closed set of $M$ for each $i$, and $f\left(V_{i}\right)$ is an open set of $M$ for each $i$. Hence, by Lemma 5 ,

Ind $X \leqslant$ Ind $M+$ ind $X=\operatorname{dim} M+$ ind $X \leqslant \operatorname{dim} X+$ ind $X$.

This completes the proof of Theorem 1 .

In the proof of Lemma 5, the following theorem is essentially proved.

THEOREM 6. $A$ paracompact $\sigma$-space with ind $X \leqslant 0$ admits a special family in the sense of Leibo [4].

REMARK. The inequality in Theorem 1 does not hold even if $X$ is compact. In fact, Filippov [9] obtained compact spaces $R_{i}, i=1,2,3, \ldots$, such that $\operatorname{dim} R_{i}=$ 1 , ind $R_{i}=i$ and Ind $R_{i}=2 i-1$. Corollary 2 is also restricted in generalization by Nagami's example [10] of a normal space $Z$ with $i n d=0, \operatorname{dim} Z=1$, Ind $Z=2$.

\section{REFERENCES}

1. C. J. R. Borges, On stratifiable spaces, Facific J. Math. 17 (1966), 1-16.

2. R. Engelking, Dimension theory, North-Holland, Amsterdam, 1978.

3. I. M. Leibo, On the equality of dimensions for closed images of metric spaces, Soviet Math. Dokl. 15 (1974), 835-839.

4. __, On closed images of metric spaces, Soviet Math. Dokl. 16 (1975), 1292-1295.

5. S. Oka, Dimension of finite unions of metric spaces, Math. Japon. 24 (1979), 351-362.

6. __ A generalization of free L-spaces, Tsukuba J. Math. (to appear).

7. A. Okuyama, Some generalizations of metric spaces, their metrization theorems and product spaces, Sci. Rep. Tokyo Kyoiku Daigaku Sect. A 9 (1967), 236-254.

8. B. A. Pasynkov, On the spectral decomposition of topological spaces, Amer. Math. Soc. Transl. (2) 73 (1966), 83-134.

9. V. V. Filippov, On bicompacta with noncoinciding inductive dimensions, Soviet Math. Dokl. 11 (1970), 635-638.

10. K. Nagami, $A$ normal space $Z$ with ind $Z=0, \operatorname{dim} Z=1$, Ind $Z=2$, J. Math. Soc. Japan 18 (1966), 158-165.

Department of Mathematics, Kanagawa University, Rokkakubashi, Kanagawa-ku, YOKOHAMA, 221 JAPAN 